\title{
THE COHESIVE GRANULAR COLLAPSE AS A CONTINUUM : PARAMETRIZATION STUDY
}

\author{
ANAIS ABRAMIAN ${ }^{1}$, LYDIE STARON ${ }^{1}$ AND PIERRE-YVES LAGREE ${ }^{3}$ \\ ${ }^{1}$ Institut d'Alembert, Sorbonne Université, CNRS \\ 4 Place Jussieu, 75252 Paris Cedex 05 \\ anais.abramian@upmc.fr
}

Key words: Granular Materials, Cohesion, Navier-Stokes solver

\begin{abstract}
Although intensive research on the flow of dry granular materials has allowed for the proposition of continuum rheology and modelling, the behaviour of flowing cohesive material has attracted less attention so far. To start modelling such cohesive flows, we first focus on the configuration of a granular collapse, which is a simple benchmark test. Specifically, we compare granular-collapse experiments of cohesive grains with numerical simulations, where we test a simple rheology for the material : the so-called $\mu(I)$-rheology, supplmented by a yield stress for cohesion. This document reports the sensitivity of our numerical simulations on the parameters of the rheology, often challenging to measure in experiments.
\end{abstract}

\section{INTRODUCTION}

Cohesive granular materials are ubiquitous in natural flows, often induced by capillary bridges of water between grains. A recent example of such a flow is the landslide of Llusco, in Peru [1] (Fig. 1). After intense rains, the soil lost cohesion and collapsed, without any seismic activity. This dramatic event reveals our misunderstanding on the static and the dynamics of a cohesive collapse.

We also frequently encounter granular materials in an industrial context, such as in construction, phar-

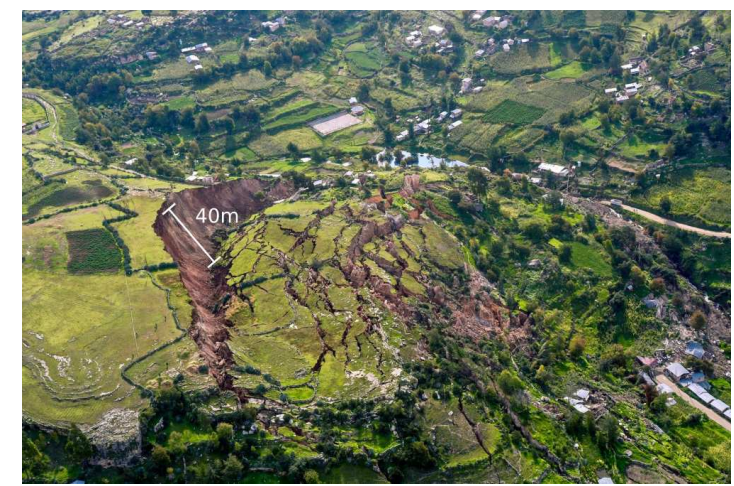

Figure 1: Picture of a landslide which occured in Llusco (Peru), in 2018. This collapse is typical of a cohesive granular flow, featuring a first fracture plane, with secondary fractures transverse to the flow. From [1]. 
maceuticals or food-processing industry. In this context, cohesive flows can clog conveyors or silos, and then stop a production chain. The need to facilitate transport and handling of powders leads the use of different industrial testers. Among different testers, the Hosokawa tester yields a "flowability" index between 0 and 100, based on a series of experiments such as the angle of repose, the tapped bulk density or the particle-size distribution. However, these measurements remain qualitative, and, even if they enable to compare two powders, they are mostly done in quasistatic conditions. Our objective is to model the dynamics of cohesive granular material and understand the physics which is behind this concept of flowability.

Although the flow of dry granular materials is now relatively well modeled by the $\mu(I)$-rheology [2], how cohesion forces affects the rheology and the flow dynamics remains elusive. Most of recent work focused on the wet granular materials, where a small amount of liquid induces cohesion forces by capillary bridges [6]. However, this cohesion force between two ideal spheres depends mainly on the shape of the bridge, and on its position, which are impossible to control experimentally.

To bypass this issue, Gans et al. (2020) [4] use a new cohesion-controlled granular material. It consists of glass beads coated by a polymer, where cohesion of the bulk increases with the polymer thickness. Consequently, the cohesion of the bulk can easily be tuned by varying the quantity of polymer during the preparation. After having characterized the onset of the flow of this new material on an inclined plane, its dynamics is now investigated in another simple configuration: the granular collapse. The material is initially prepared in a column of a given aspect ratio $a=H / L$ [Fig. 2(a)]. At the beginning of the experiment $(t=0)$, the gate is removed and the column collapses under its own weight.
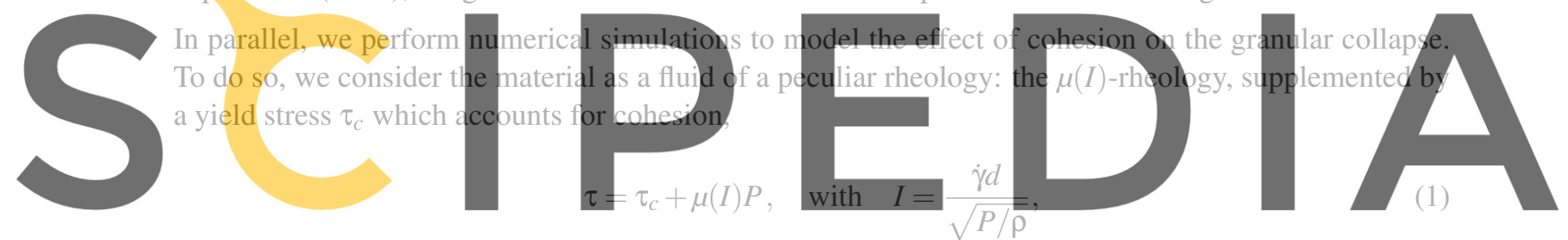

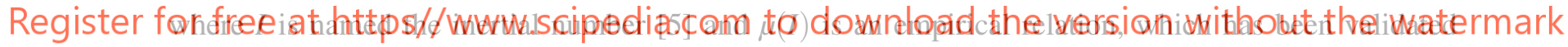
experimentally and numerically for dry granular materials $[3,7]$ :

$$
\mu(I)=\mu_{s}+\frac{\mu_{2}-\mu_{s}}{I_{0} / I+1}
$$

with $\mu_{s}$ the friction coefficient, and $I_{0}, \Delta \mu=\mu_{2}-\mu_{s}$ are empirical parameters. The friction coefficient $\mu_{s}$ has been measured experimentally through the inclined-plane configuration, and is constant with the cohesion force, equal to $\mu_{s}=0.4 \pm 0.05$ with these beads. However, the parameters $I_{0}$ and $\Delta \mu$ are difficult to measure experimentally. First, $\mu_{2}$ is the friction coefficient between two grains only, which requires small-scale experiments. Then, the measurement of $I_{0}$ can only be fitted on the whole rheology, which needs a home-made shear cell, with no-slip conditions on the walls, while the confinement pressure $P$ can vary. This makes a challenging apparatus.

Consequently, we focus here on the influence of these two parameters $\left(I_{0}\right.$ and $\left.\Delta \mu=\mu_{2}-\mu_{s}\right)$ in the numerical resolution of the cohesive granular collapse. Specifcally, we investigate their influence on the velocity of the collapse, and on the length of the final deposit (or final runout). By comparing these results with the experiments, we demonstrate that the dependency of the rhology on $I$ is necessary to 

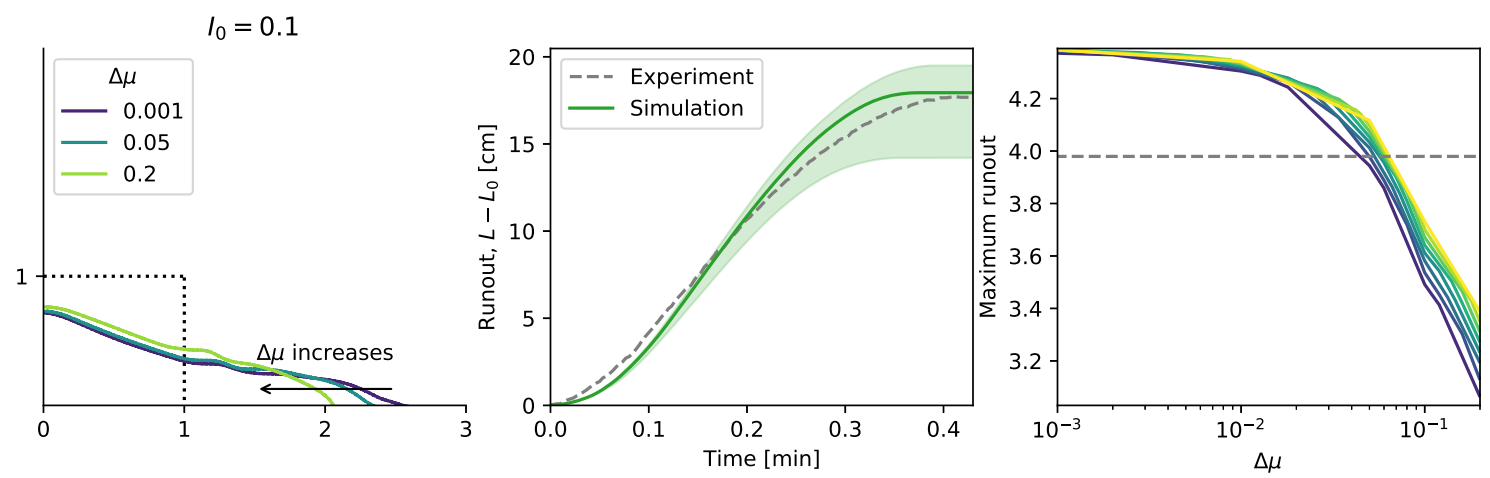

Figure 2: Variation of $\Delta \mu$. (a) Final shape of the deposit as a function of $\Delta \mu$ for a fixed $I_{0}$. (b) Comparison between an experiment and the corresponding numerical simulation. The upper bound of the green area is $\Delta \mu=0$. The lower bound is $\Delta \mu=0.4$. (c) Variation of the final runout as a function of $\Delta \mu$ for different $I_{0}$.

model the collapse correctly. We show, however, that our uncertainty on the rheological parameters can lead about $20 \%$ of uncertainty on the final runout.

\section{SENSITIVITY TO $\Delta \mu$}

A first parameter involved in the granular rheology (1) but not measured experimentally is $\Delta \mu=\mu_{2}-\mu_{s}$.

It is the friction difference

it is the friction difference

experiments of Jop et $a$.

diameter $d=0.53 \mathrm{~mm}$.

Here, we vary this parameter andstudy its influence
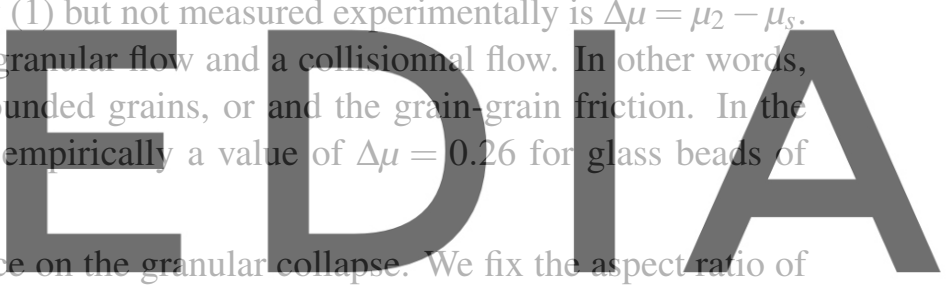

the column to $a=1$, and set the cohesion through a characteristic length $: \ell_{c}=\tau_{c} / \rho g=3.6 \mathrm{~cm}$. Fig.

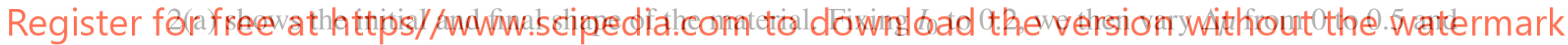
investigate its influence on the dynamics of the collapse, and on the final shape of the deposit.

After the column fiows, the material ends up with a static final shape, featuring a characteristic bump, which was not sheared during the collapse [Fig. 2(a)]. The value of $\Delta \mu$ qualitatively modifies this final shape as follow. The angle of the front increases when $\Delta \mu$ increases, and the bump is more pronounced as $\Delta \mu$ is higher. This makes the position of the front particularly sensitive to $\Delta \mu$.

To investigate this sensitivity, plot the time-evolution of the front's position, substracted by the initial length of the column, $L-L_{i}$, for $\Delta \mu=0.2$ [Green line, Fig. 2(b)]. We then compare this numerical result to an experiment in the same configuration [Dashed grey line, Fig. 2(b)].

Now, we vary $\Delta \mu$ from zero to 0.4 [Green area, Fig. 2(b)]. We observe a qualitatively good agreement, although it is better at the beginning of the collapse than at the end. The final runout is more sensitive to $\Delta \mu$, than its derivative, which corresponds to the velocity of the collapse. When $\Delta \mu$ is small enough, the final runout is the higher. When $\Delta \mu$ increases, the final runout decreases. Finally, this gives an uncertainty of $25 \%$ on the final length of the deposit.

Overall, the variation of the final runout with $\Delta \mu$ is shown on Fig. 2(c). Each line, which corresponds to 

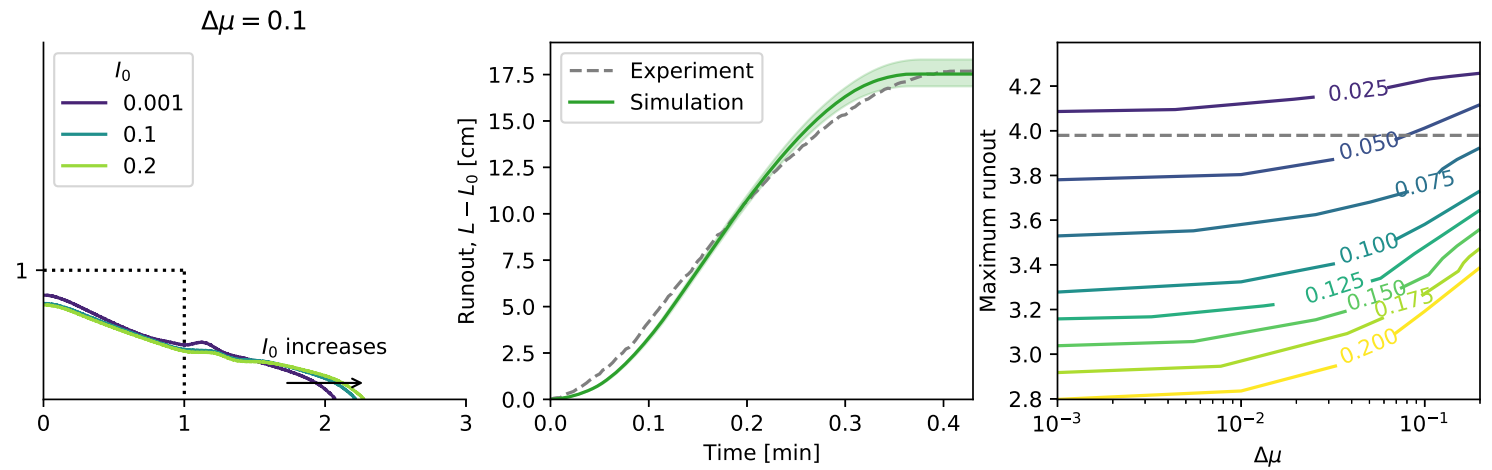

Figure 3: Variation of $I_{0}$. (a) Final shape of the deposit as a function of $I_{0}$ for a fixed $\Delta \mu$. (b) Comparison between an experiment and the corresponding numerical simulation. The upper bound of the green area is $I_{0}=0.6$. The lower bound is $I_{0}=0.1$. (c) Variation of the final runout as a function of $I_{0}$.

a different value of $I_{0}$, are close. Thus, the parameter $\Delta \mu$ seems to control the most the final runout of the collapse, at least more than $I_{0}$. To confirm it, we perform a detailed investigation of the influence of the parameter $I_{0}$ in the next section.

3 SENSITIVITY TO $I_{0}$

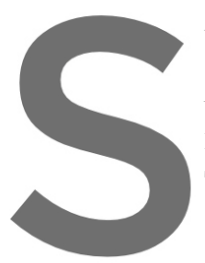

We now test the sensitivity
$I_{0}$. This parameter is usuall
measurements. Jop et al. (
To investigate the influenc
same procedure than previ
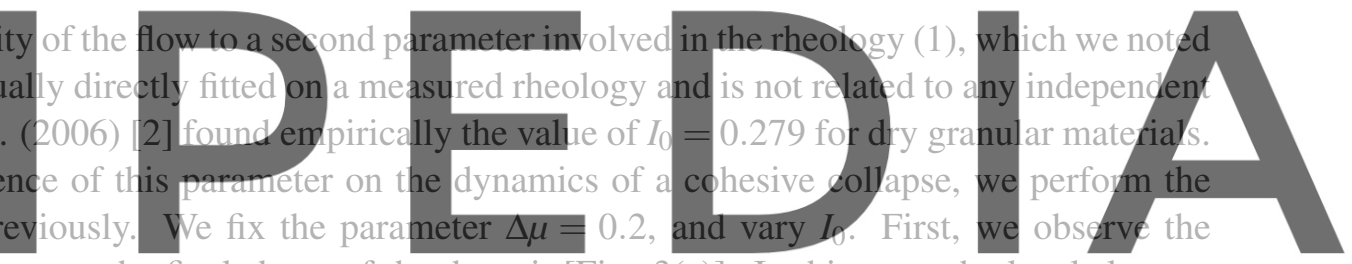

influence of this parameter on the final shape of the deposit [Fig. 3(a)]. In this case, the local slope at

Register for front is nt modified, but thisparangeter makes the scale of the inertial number vary This reduces the size of the bump, and therefore the final runout, although slightly.

To measure quantitatively this influence on the final runout, we plot the position of the front as a function of time for the same configuration and compare it to the experiment [Fig. 3(b)]. By varying $I_{0}$ from $10^{-3}$ to 0.4 , we show the variation of this function as $I_{0}$ increases. Once again, the velocity of the collapse is less sensitive than the final runout, which can vary of about $10 \%$ in the range of values we explored. Fig. 3(c) shows the dependency of the final runout as a function of $I_{0}$ for different $\Delta \mu$. Here again, this shows that it is $\Delta \mu$ which is most important in the choice of the parameters. In particular, we superimposed the value of the final runout measured experimentally for the same configuration [dashed grey line, Fig. 3(c)], and deduce that a large range of values for the two parameters $I_{0}$ and $\Delta \mu$ are possible to fit with the final runout.

In the next section, we explore the range fo both parameters together for the runout and the velocity and investigate which couple of values fits the experimental data at best. 

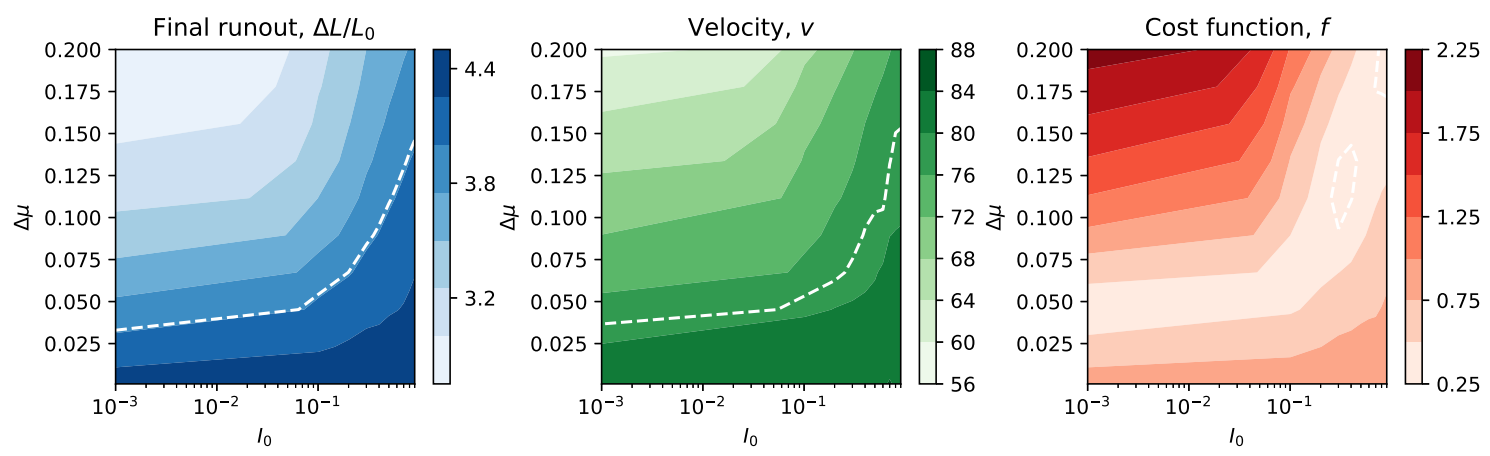

Figure 4: (a) Map of runout. Dashed white line: experimental value of the final runout. (b) Map of velocity. Dashed white line: experimental value of the final runout. (c) Map of cost function. Dashed white line: minimization of the cost function.

\section{SENSITIVITY TO BOTH PARAMETERS}

We now vary sequentially $I_{0}$ and $\Delta \mu$, and compute the corresponding final runout of the collapse. We can then plot the final runout as a function of the two parameters $\Delta \mu$ and $I_{0}$ [given by the colorbar, Fig. 4(a)]. On this map, we superimpose the experimental value, and find a few couples of parameters which could fit at best the final runout [white dashed line, Fig. 4(a)].

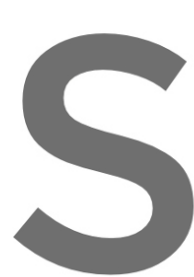
However, it is not bec

collapse is robust. Indeed, Consequently, we also

front position, as a function

find the best fit for approx

To account for the entire dynamics of the collapse,
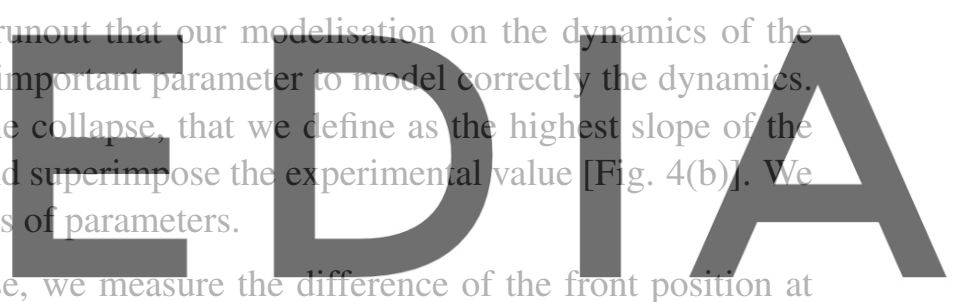

each time, for the experiment and the numerical simulation. In other words, we define a cost function $f$,

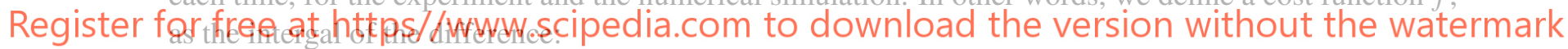

$$
f=\int\left(r_{\text {num }}-r_{\exp }\right)^{2} d t
$$

where $r=\Delta L / L_{0}$ is the normalized runout as a function of time. We now want to minimize this function to fit the experiments at best.

We thus plot this function $f$ for both parameters [Fig. 4(c)]. The minimum value of this function is the dashed white line, and corresponds to the couple of parameters : $I_{0}=0.35$ and $\Delta \mu=0.125$. Still, these values are in this range of parameters we explored. We must compare these values with the dry granular materials.

Finally, to avoid dealing with two parameters, we can linearize the rheology and make it vary through one single parameter to facilitate the study, which we do in the next section. 

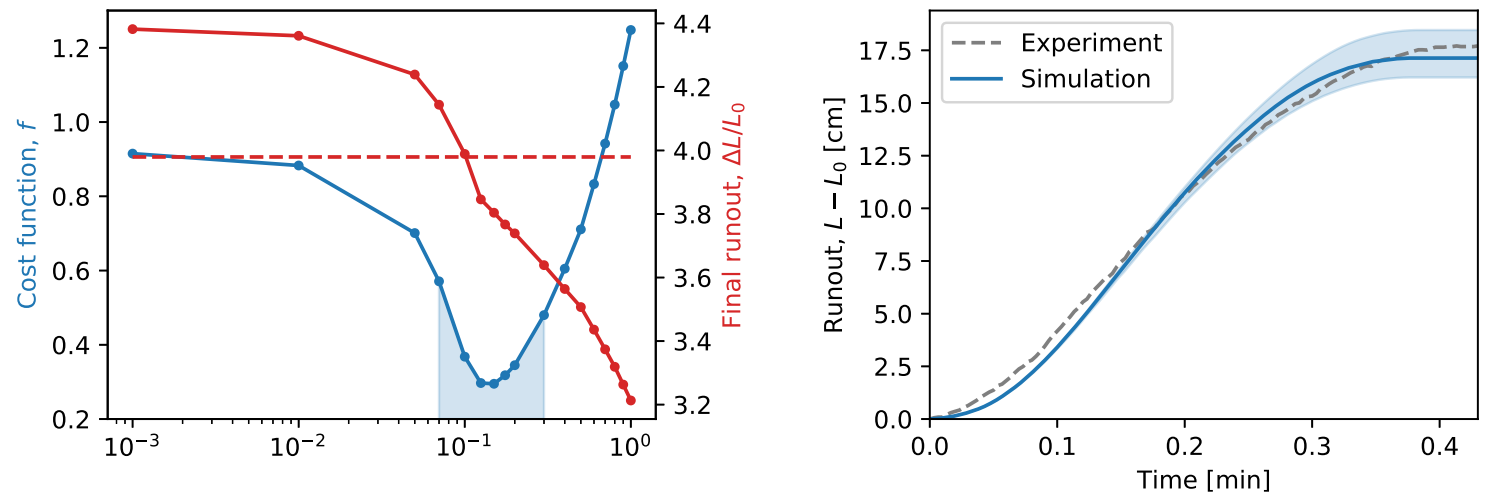

Figure 5: (a) In blue: cost function $f$ defined by equation (3) as a function of parameter $a$ involved in the linearized rheology (4). In red: final runout of the deposit as a function of $a$. Dashed blue line : experimental value of the final runout. (b) Position of the front as a function of time. Blue: numerical simulation for $a=0.125$. Dashed grey line : experimental data. The blue area corresponds to the range of $a$ colored on the panel (a).

\section{LINEARIZED RHEOLOGY}

For columns of small aspect ratios, we can linearize the rheology on the inertial number, through $\mu(I)$ as follows:
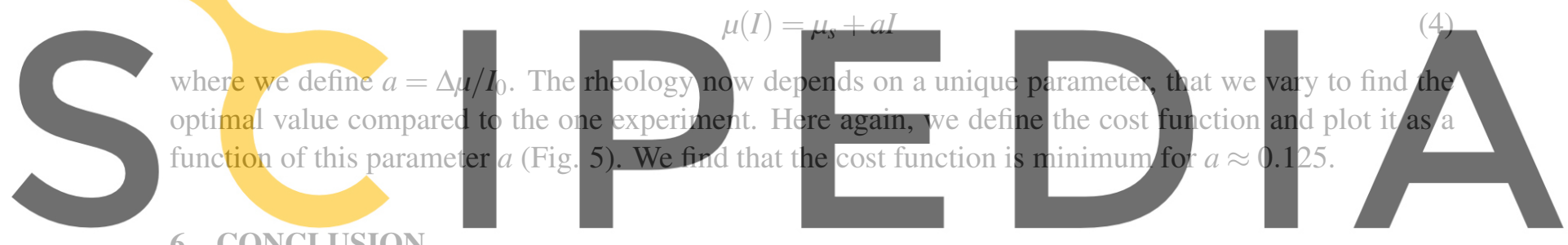

6 CONCLUSION

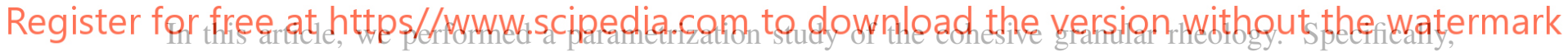

we investigated the influence of two parameters involved in the rhology : $\Delta \mu$ and $I_{0}$. We observed that

$\Delta \mu$ infiuences the most the finai shape of the deposit, and in particuiar its final runout. We found a few couples of values which fit the experimental runout and velocity at best. Finally, we linearized the rheology, and found in this case a unique value of parameter which fits at best the experiments :

$$
\mu(I)=\mu_{s}+a I
$$

where $a \approx 0.125$.

This could be generalized to other cases of cohesion and aspect ratios, but this study gives, at least, insights on the parameters involved in the rheology and on the amplitude of our uncertainties.

\section{REFERENCES}

[1] Roca Guevara, Nicole Katherine. "Impacto Ambiental por deslizamiento de suelo en el Sector Campanayocpata del Centro Poblado de Lutto en Chumbivilcas-Cusco 2018.” (2018). 
[2] Jop, P., Forterre, Y., \& Pouliquen, O. (2006). A constitutive law for dense granular flows. Nature, 441(7094), 727.

[3] Lacaze, L., \& Kerswell, R. R. (2009). Axisymmetric granular collapse: a transient 3D flow test of viscoplasticity. Physical Review Letters, 102(10), 108305.

[4] Gans, A., Pouliquen, O., and Nicolas, M. (2019). A cohesion-controlled granular material. Physical Review E 101.3 (2020): 032904.

[5] GDR MiDi 2004 (2004), On dense granular flows, Eur. Phys. J. E 14, 341-365

[6] Bocquet, L., Charlaix, E., \& Restagno, F. (2002). Physics of humid granular media. Comptes Rendus Physique, 3(2), 207-215.

[7] Lagrée P.-Y., L. Staron \& S. Popinet, The granular column collapse as a continuum: validity of a two-dimensional Navier-Stokes model with the mu(I) rheology, J. Fluid Mech. vol 686, pp 378-408 (2011)
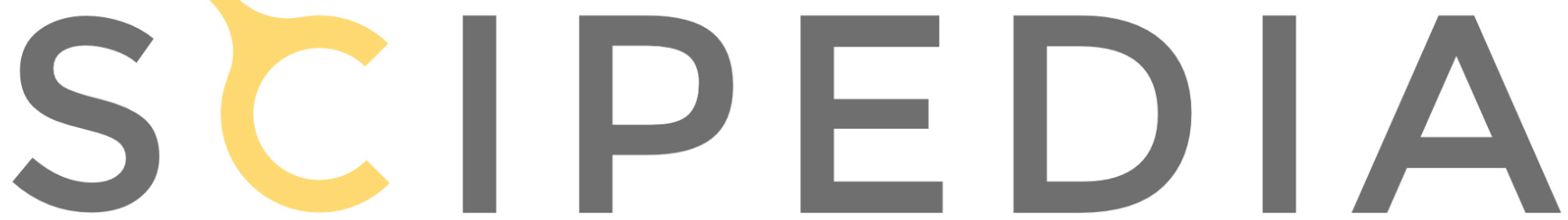

Register for free at https//www.scipedia.com to download the version without the watermark 